\title{
Identifying Ideal Brow Vector Position: Empirical Analysis of Three Brow Archetypes
}

\author{
Ashley A. Hamamoto, BS ${ }^{1}$ Tiffany W. Liu, BS, BA ${ }^{1}$ \\ ${ }^{1}$ Beckman Laser Institute, University of California, Irvine, Irvine, \\ California \\ ${ }^{2}$ Department of Otolaryngology-Head and Neck Surgery, University \\ of California, Irvine, Irvine, California \\ ${ }^{3}$ Department of Biomedical Engineering, University of California, \\ Irvine, Irvine, California
}

Brian J. Wong, MD, $\mathrm{PhD}^{1,2,3}$

Facial Plast Surg 2013;29:76-82.

\begin{abstract}
Address for correspondence and reprint requests Brian J. Wong, MD, PhD, Beckman Laser Institute, 1002 Health Sciences Rd., Irvine, California 92617 (e-mail: bjwong@uci.edu).
\end{abstract}

\section{Abstract}

\section{Keywords}

- brow position

- browplasty

- brow aesthetics

- eyebrow methods

- browlift
Background Surgical browlifts counteract the effects of aging, correct ptosis, and optimize forehead aesthetics. While surgeons have control over brow shape, the metrics defining ideal brow shape are subjective.

Objectives This study aims to empirically determine whether three expert brow design strategies are aesthetically equivalent by using expert focus group analysis and relating these findings to brow surgery.

Methods Comprehensive literature search identified three dominant brow design methods (Westmore, Lamas and Anastasia) that are heavily cited, referenced or internationally recognized in either medical literature or by the lay media. Using their respective guidelines, brow shape was modified for 10 synthetic female faces, yielding 30 images. A focus group of 50 professional makeup artists ranked the three images for each of the 10 faces to generate ordinal attractiveness scores.

Results The contemporary methods employed by Anastasia and Lamas produce a brow arch more lateral than Westmore's classic method. Although the more laterally located brow arch is considered the current trend in facial aesthetics, this style was not empirically supported. No single method was consistently rated most or least attractive by the focus group, and no significant difference in attractiveness score for the different methods was observed ( $p=0.2454)$.

Conclusion Although each method of brow placement has been promoted as the "best" approach, no single brow design method achieved statistical significance in optimizing attractiveness. Each can be used effectively as a guide in designing eyebrow shape during browlift procedures, making it possible to use the three methods interchangeably.
Eyebrows play a significant role in an individual's expression of emotions and nonverbal communications. ${ }^{1}$ Some consider the brow line as the master line of the face, serving as the reference to which other angles and contours of the face are aligned. ${ }^{2}$ Given the importance of brows to an individual's appearance, browlift procedures are performed to counteract the effects of aging (i.e., brow ptosis) and to optimize facial aesthetics. By creating a pull along particular vectors, sur- geons have control over eyebrow shape (peak, tail, and overall geometry), height, and distance from the orbital rim. This is accomplished via different surgical methods including direct, midforehead, trichophytic, endoscopic, and coronal browlifts and nonsurgical techniques such as the precise use of injectable neuromodulators.

Among makeup artists, there are several competing methods for ideal eyebrow design. Three stand out as they can be
Issue Theme Aesthetic Treatment of the Eyelids and Midface; Guest Editors, Michael M. Kim, MD, and Tom D. Wang, MD, FACS
Copyright $\odot 2013$ by Thieme Medical Publishers, Inc., 333 Seventh Avenue, New York, NY 10001, USA. Tel: +1(212) 584-4662.
DOI http://dx.doi.org/ 10.1055/s-0033-1333841. ISSN 0736-6825. 


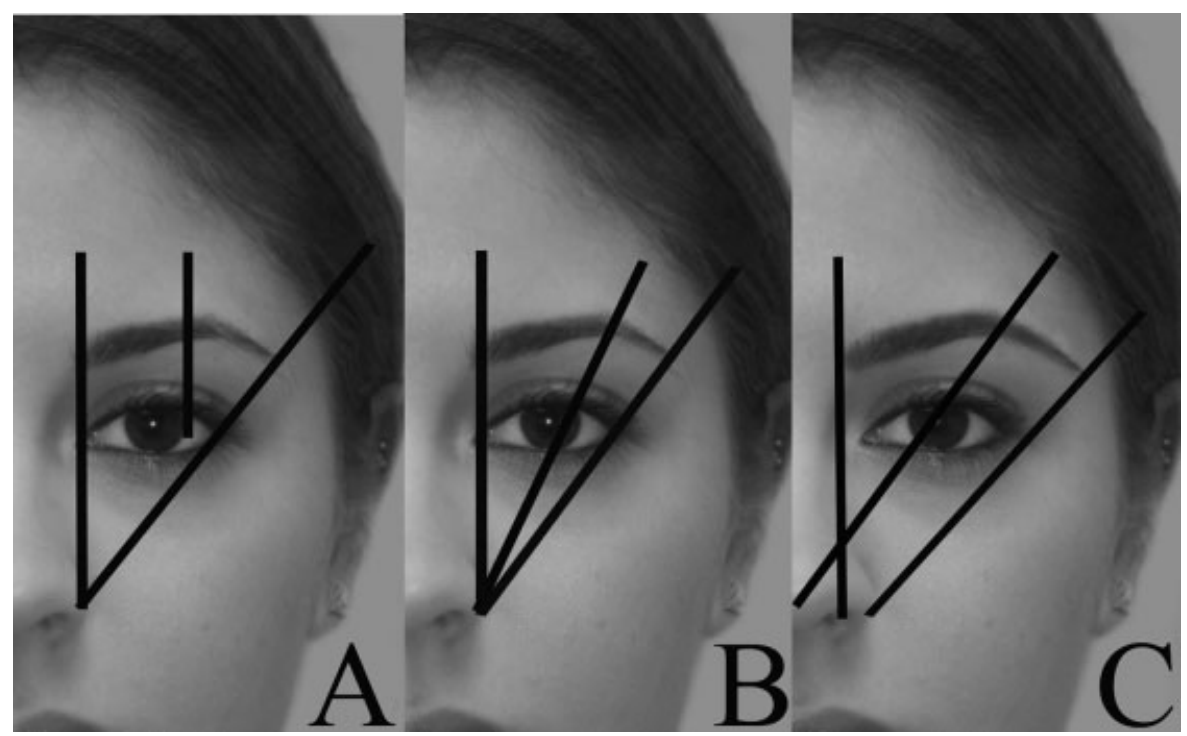

Fig. 1 The three selected eyebrow design methods: Westmore (A), Lamas (B), and Anastasia (C). In each image, lines determine the ideal locations of the beginning, the arch, and end of the brow.

replicated with limited subjective input from the artist and are canonical and subject to limited interpretation. Westmore's approach is most heavily cited in medical literature, and many have commented on his general strategy. ${ }^{3} \mathrm{He}$ contends that the brow should start on the same vertical plane as the lateral extent of the nasal ala and the inner canthus, arch perpendicular to the lateral limbus, and end on an oblique line drawn from the most lateral point of the nasal ala through the lateral canthus ( - Fig. 1a). Brennan ${ }^{4}$ modified Westmore's approach and suggested that the eyebrow should start along an imaginary vertical line drawn from the ala of the nose to the brow line, the brow should arch above the supraorbital rim, and the brow should end on an imaginary oblique line drawn from the ala of the nose to the lateral canthus. Angres ${ }^{5}$ said the location of the brow arch depends on the distance between an individual's eyes such that the arch for well-spaced eyes should be located above the pupil, above the lateral limbs as Westmore's method if the eyes are close-set, and located nasal to the medial limbus for wide-set eyes. According to Cook et al, ${ }^{6}$ the arch should be placed more laterally above the lateral canthus to prevent imparting an unnatural, surprised look.

Lamas, ${ }^{7}$ a licensed cosmetologist trained under Vidal Sassoon's Paul Mitchell, asserts that the brow should begin on a perpendicular line drawn from the base of the nose to the brow line, arch on an imagined line extending from the tip of the nose to the lateral border of the iris, and end on a diagonal line drawn from the base of the nose tangent to the corner of the eye ( $\mathbf{- F i g . ~ 1 b )}$ ). Anastasia, ${ }^{8}$ a celebrated Beverly Hills brow expert, contends that the brow starts on a perpendicular line drawn from the middle of the nostril, arches on a line drawn from the center of the nose through the center of the pupil, and ends on a line drawn from the edge of the corresponding nasal ala through the outer edge of the eye (-Fig. 1c).

Evidence-based research on brow position and shape has been rigorously studied and examined in an effort to establish a systematic approach to defining the ideal brow position. Gunter and Antrobus referenced the study by Matarasso et $\mathrm{al}^{3}$ that proposed the distance from the brow to the eyelid crease should be $1.6 \mathrm{~cm}$, and the distance from the eyebrow to midpupil should be $2.5 \mathrm{~cm}$. The study reported that the brow should be $1 \mathrm{~cm}$ from the eyebrow to the supraorbital rim and $\sim 5$ to $6 \mathrm{~cm}$ from the eyebrow to the hairline. ${ }^{3}$ Sclafani and Jung ${ }^{9}$ investigated optimal brow position by asking volunteers to self-position their idea of an optimal brow. They found that female subjects preferred: (1) the brow tail to be slightly below the medial brow terminus; (2) the midpupillary line, brow peak, and lateral canthal brow to be placed higher than the medial canthal brow; and (3) the brow peak to be $\sim 13 \mathrm{~mm}$ above the inferior border of the medial brow. Another study researched the impact of age on an individual's perception of ideal brow design. Biller and $\mathrm{Kim}^{10}$ found that age impacts the location of the ideal brow apex; specifically, they found that younger participants preferred a more lateral brow apex and older participants preferred a more medial apex.

By evaluating existing brow design systems (Westmore, Lamas, Anastasia), this study sought to investigate whether a single ideal brow design strategy exists to provide surgeons with a better understanding of ideal brow shape and design for application in aesthetic browlift procedures where brow position and geometry can be controlled. Using the guidelines from these three methods, brow shape was digitally modified for 10 female faces with the resultant changes in brow shape ranked for attractiveness by a cohort of professional makeup artists.

\section{Methods}

A comprehensive literature search using search engines, online databases (e.g., PubMed), and the World Wide Web dating back to 1974 yielded over 100 medical journal articles, lay articles, and Web sites committed to the latest eyebrow 


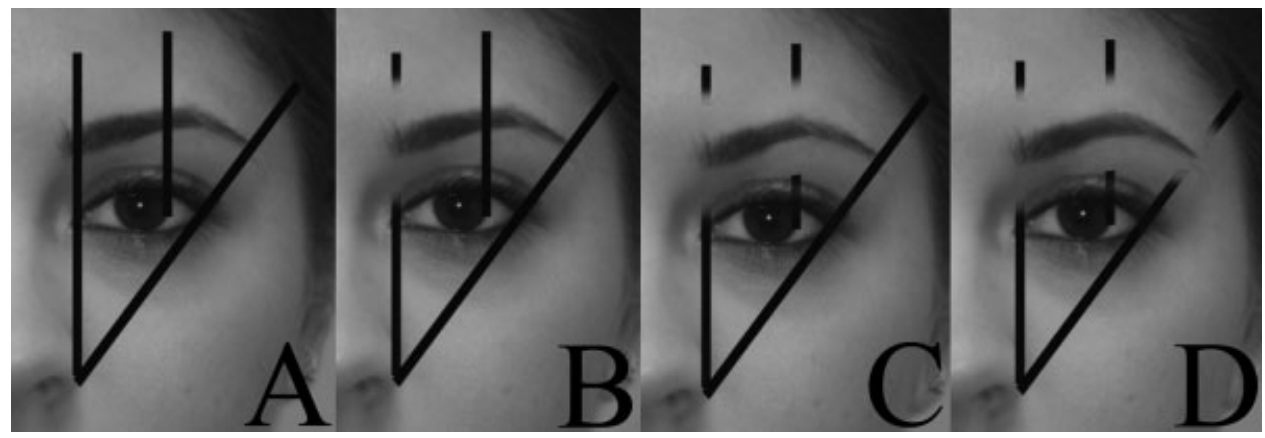

Fig. 2 (A to C) Step-by-step process of applying Westmore's brow technique onto a face. (A) Insertion of guidelines by creating a separate layer within Photoshop to determine the location of the ideal start, arch, and end of the brows. (B) Alteration of the start of the brow, brow arch (C), and end of the brow (D). (D) Example of a left brow that has completed alteration. To alter the start of the brow, two copies of the original photograph were opened: one image contained the black guidelines and the other did not. A copy of the start of the brow was made from the image without guidelines and pasted in the image with guidelines based on the guidelines. The history brush function was used to blend in the new start of the brow to make the alteration realistic. This methodology was repeated for the arch and end of the brow. In the final image, the black lines were removed by deleting that respective layer.

fashions and trends. Key search terms included: "eyebrow methods," "ideal eyebrow," "position of the eyebrow," "eyebrow position," "eyebrow height," "surgery eyebrow methods," "eyebrow surgery," "eyebrow vectors," "eyebrow peak," "eyebrow vector position," "brow ptosis position," "desired brow position," "cosmetology brow position," "makeup brow position," "desired shape of the brow," "desired brow for surgery," "brow elevation," "celebrity brow artists," "professional brow artist," and "brow cosmetologist."

From the literature search, brow design methods of Westmore, Lamas, and Anastasia were selected because they presented detailed, replicable methods and diagrams for recreating their ideal brow designs. Alternative methods were rejected because they provided vague descriptions without complete guidelines or diagrams depicting their approach, leaving the methods open to significant subjective interpretations.

All three methods ( $\boldsymbol{- F i g . 1} \mathbf{1}$ a to c) share common features of modified arch position and slender tail. However, the brow arch position (located along a medial to lateral axis) varied among the three methods and currently remains the primary point of controversy for brow designs.

Digital photographs of 250 female, Caucasian volunteers between the ages of 18 to 25 were taken with the approval of the Institutional Review Board at the University of California, Irvine. Photographs were morphed using Morphman 2000 (STOIK Imaging, LTD, Moscow, Russia) to create a synthetic facial image that was a 50:50 morph of the two original images. Adobe Photoshop CS 4 (Adobe, San Jose, CA) was used to make the image more realistic by removing artifacts produced by the morphing process. In total, a database of 300 synthetic faces was constructed. Details of our use of synthetic facial images have been previously reported. ${ }^{11}$

From this synthetic database, 10 faces with realistic facial characteristics were selected based on brow and facial symmetry. Photoshop was used to alter the 10 selected faces in accordance with each of the three brow design methods ( - Fig. 1a to c). For Westmore (-Fig. 2a to d) and Lamas' ( - Fig. 3a to d) methods, lines were drawn on each image in a separate layer in Photoshop to determine the ideal start, arch,

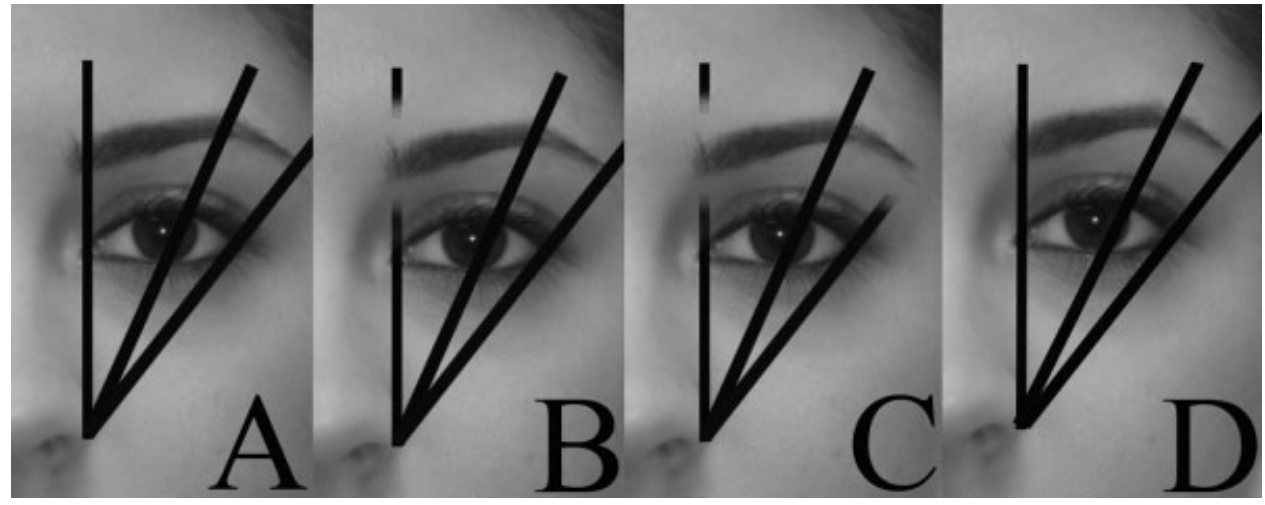

Fig. 3 (A to C) Step-by-step process of applying Lamas' brow technique onto a face. (A) Insertion of guidelines to determine the location of the ideal start, arch, and end of the brows. (B) Alteration of the medial end of the brow, brow arch (C), and end of the brow (D). (D) Example of a left brow that has completed alteration. To alter the start of the brow, two copies of the original photograph were opened: one image contained the black guidelines and the other did not. A copy of the start of the brow was made from the image without guidelines and pasted in the image with guidelines based on the guidelines. The history brush function was used to blend in the new start of the brow to make the alteration realistic. This methodology was repeated for the arch and end of the brow. In the final image, the black lines were removed by deleting that respective layer. 


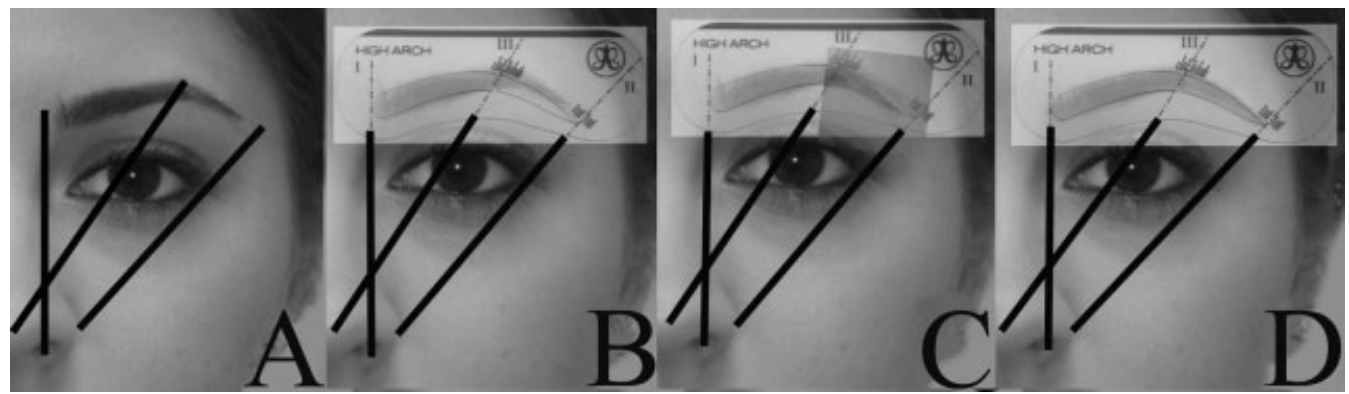

Fig. 4 (A to C) Step-by-step process of applying Anastasia's brow technique onto a face. (A) Stencil placement and alteration of the start of the brow into the brow arch. Alterations of part of (B) the brow arch and (C) the end of the brow. (D) Example of a completely altered left brow. To alter the images, the stencil was first placed on the face. Note that Anastasia's kit offered various stencils (i.e., petite arch, slim arch, medium arch, high arch, and full arch). An Anastasia brow expert was asked to help select the best stencil for each of the 10 faces (Donna Mee, personal communication, February 15, 2010). Next, two copies of the original photograph were opened: one image containing the stencil and the other without. A copy of the start of the brow was made from the image without the stencil and pasted in the image with stencil based on the stencil. The history brush function was used to blend in the new start of the brow to make the alteration realistic. This methodology was repeated for the arch and end of the brow. In the final image, the stencil was removed by deleting its respective layer.

and end of the brow locations, and then the brows were modified in accordance. After, the lines were erased. In contrast, Anastasia's method utilizes stencils available for purchase commercially. After purchasing, Anastasia's stencils were scanned, appropriately scaled in Photoshop and used as the guideline for recreating her brow design ( - Fig. $4 a$ to $\mathbf{d}$ ). All brow images altered in compliance with Anastasia's technique were separately evaluated by two Anastasia eyebrow artists located at two separate malls in Orange County, California. A total of 30 modified images were produced and high-quality, glossy colored hard copies were generated. Fifty professional makeup artists from retail department stores (Nordstrom, Macy's, and Bloomingdales) at upscale shopping centers (South Coast Plaza, Irvine Spectrum, and Fashion Island) in Orange County, California were asked to rank the attractiveness of the three-image faces relative to each other for each of the 10 faces. Analysis of variance was performed using Matlab ${ }^{\circledR}$ (Mathworks, Natick, MA) to conduct a Student's $t$ test to evaluate the makeup artists' ordinal ratings to determine if there is a difference between these three brow design methods. A $p$ value of 0.05 was determined to be significant.

\section{Results}

The $t$ test yielded a $p$ value of 0.2454 , showing no significant difference observed between the ordinal ranking for the three different brow placement methods; no single method was consistently rated as most or least attractive (-Fig. 5). Each method ranked most and least attractive for at least one face. - Fig. 6 illustrates the similarity in overall average scores for the three methods with standard errors of $0.0787,0.0767$, and 0.0784 for Westmore, Lamas, and Anastasia, respectively. This shows no significant difference between methods. The contemporary methods of Anastasia and Lamas place the brow arch more lateral than the more classic method of

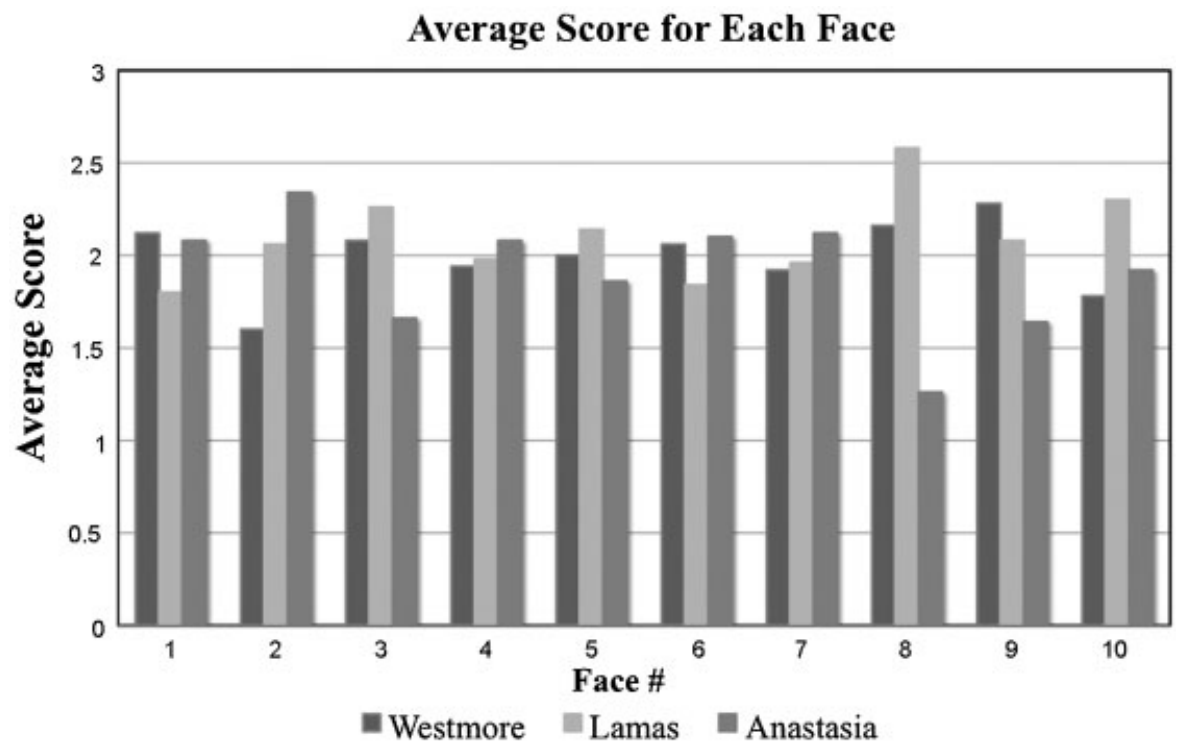

Fig. 5 The ordinal rank for each face for each method. 


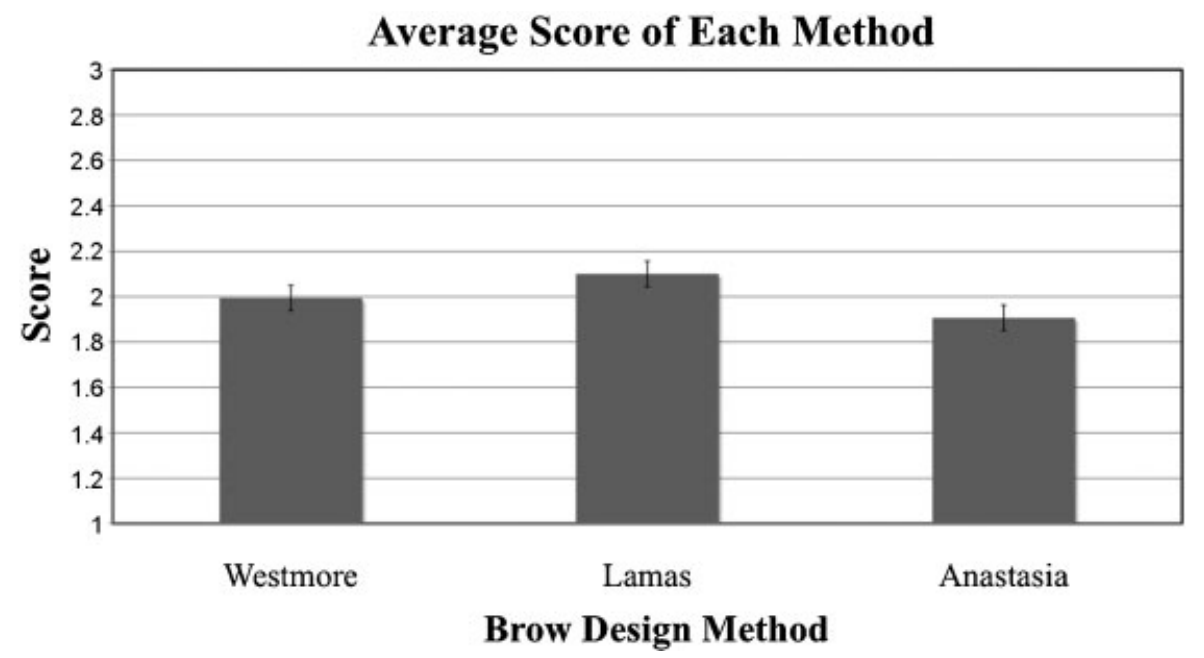

Fig. 6 Average ordinal rank for each brow design method.

Westmore. Although a more laterally located brow arch is considered the current trend by both cosmetic surgeons and cosmetologists, this style was not supported empirically.

\section{Discussion}

Geometric analysis of eyebrow shape is complicated by the multiple variables of dimensional metrology and anatomy. The complexities of human aesthetic preference coupled with the lack of empiric standard measurement techniques for the brow makes finding the ideal brow shape a difficult, multidimensional problem. Depending on which vectors are pulled, surgeons can control brow shape (peak, tail, and overall geometry), as well as height and distance from orbital rim; however, no verified guidelines exist for these surgical alterations. The scientific basis for eyebrow-shaping methods remains unclear, even for the most heavily studied and referenced method in medical literature-Westmore's method. Thus our study aimed to determine if an ideal brow design method exists by evaluating expert eyebrow design techniques in the attempt to establish an ideal vector positioning of the brow as a practical solution to this multidimensional problem of brow shape and placement.

Although the three methods selected for this study share common features of enhanced arch position, slender tail, and superior elevation, they vary in start, arch, and end positions. The primary difference between the three methods is brow arch, over which a surgeon can exert modest control through most browlift operations. Despite differences in brow peak location, the study showed no statistically significant difference among the three methods. Furthermore, when makeup artists were asked how they determined the most aesthetically pleasing brow, their responses were biased toward their personal opinions and not by any one method.

The lack of statistically significant difference between the methods could be attributed to the application of Photoshop to alter the images. In Photoshop, the facial images are twodimensional, as opposed to the three-dimensional structure of a real face. Furthermore, it is difficult to recreate realistic eyebrow hair texture on the altered images, which could lead to artifacts and bias scores. Likewise, our validated approach of using synthetic images has a shortcoming: although extremely realistic as a facial simulacrum, these were digital composites/morphs of young, Caucasian women. Young women do not have browlifts, and the features associated with the aging face that are enhanced by browlifting are very different in the older patient. Obviously, correcting brow ptosis impacts upper lid anatomy as well. Finally, the basis population for this study was derived from women of European ancestry. This was due to the fact that the database we utilized to obtain digital composites/morphs only contained photos of young, Caucasian women. Race and facial shapes are other issues that can be explored, but beyond the scope of this study as well.

Aspects of the study's focus group, such as its size and composition, may also be limiting factors. Composed of 50 scorers, the focus group had a narrow scope. Its membership was also restricted by profession because only makeup artists and cosmetologists were asked to rank the images, rather than the general population. Makeup artists may be biased toward a certain method due to individual training. However, this specialized training that may produce bias also qualifies this demographic for detail-oriented analysis of faces, necessary for their professional skills in evaluating and enhancing facial aesthetics. Such a perspective gives added value to their input.

Although superficially dogmatic, judging all three brow methods still required the makeup artists to exercise their artistic judgment, entrenching an element of subjectivity into the study. For example, in conversations with several Anastasia eyebrow artists $(n=3)$, they revealed that deviating from the guideline was necessary if their own personal expertise was required (i.e., shortening or elongating the brow length). Many cosmetologists assert that one determines where the brows should start based on how far apart the individual's eyes arefor instance, starting the brows farther apart for close-set eyes to make the eyes appear further apart. However, sometimes the distance between the eyes appears greater than it actually is due to facial features such as pinched nasal tip, high nasal 
dorsum, and dark circles under the eyes and in the medial portion of the eyes. Under these circumstances, artistic judgment is exercised (Donna Mee, personal communication, February 15, 2010).

In practice, makeup artists, like surgeons, use rules as a starting point but ultimately make intuitive decisions based upon an integrated aesthetic sense of judgment. However, although an aesthetician or cosmetologist may shape or design dozens of brows per week, a surgeon will perform only a handful of forehead lifts in the same time interval. In addition, the changes a makeup artist makes are temporary due to hair regrowth, and outcomes are enhanced with cosmetics. In contrast, the changes a surgeon produces are definitive. Because multiple definitions of beauty exist, guidelines are needed for surgeons to reduce subjective input. Our data suggest that the arch can be placed anywhere between a perpendicular line drawn from the lateral limbus to a slant line drawn from the base of the nose tangent to the lateral aspect of the iris. The brow can start from a perpendicular line drawn from the middle of the nostril to the vertical plane as the lateral extent of the ala and inner canthus. The brow should end between the most lateral point of the ala through the lateral canthus and a line drawn from the edge of the corresponding nostril through lateral canthus.

Although Westmore's technique still stands the test of time, the techniques of Lamas and Anastasia are still valuable. Nevertheless, the lack of a dominant method shows that there is no statistically superior brow design technique among the three. In essence, it would be possible to use the three methods discussed in this study interchangeably in surgery.

\section{Conclusion}

A surgeon's understanding of how to optimize brow design is important for brow ptosis or rejuvenation procedures. An awareness of brow aesthetics can be applied to impact the overall outcome of a procedure. This study aimed to provide a better understanding of ideal brow shape and design, which will have application in aesthetic browlift procedures in which surgeons can control brow position and geometry.
Although each method of brow placement (Westmore, Lamas, and Anastasia) has been acclaimed as the "best" approach, none achieved statistical significance in optimizing attractiveness. Thus it is possible to use any of these three methods interchangeably in surgery without deleterious effects on the aesthetic outcomes.

\section{Acknowledgments}

Special acknowledgments go to: Amanda Lim, MD; Cyrus Manuel, BS; David Avila, BS; Davin Chark, MS, MD; Donna Mee; Ed Wu, MD; Karam Badran, MD; Lauren Tracey, MD; Michael Broukhim, DO; Natalie Popenko, BS; and Zlatko Devcic, MD.

\section{References}

1 Sadr J, Jarudi I, Sinha P. The role of eyebrows in face recognition. Perception 2003;32:285-293

2 Baker SB, Dayan JH, Crane A, Kim S. The influence of brow shape on the perception of facial form and brow aesthetics. Plast Reconstr Surg 2007;119:2240-2247

3 Gunter JP, Antrobus SD. Aesthetic analysis of the eyebrows. Plast Reconstr Surg 1997;99:1808-1816

4 Brennan HG. Correction of the ptotic brow. Otolaryngol Clin North Am 1980;13:265-273

5 Roth JM, Metzinger SE. Quantifying the arch position of the female eyebrow. Arch Facial Plast Surg 2003;5:235-239

6 Cook TA, Brownrigg PJ, Wang TD, Quatela VC. The versatile midforehead browlift. Arch Otolaryngol Head Neck Surg 1989;115: 163-168

7 Lamas P, Pemberton C. Eyebrows 101. 2007. Available at: http:// www.lamasbeauty.com/beauty/november00/eyebrows-101.htm

8 Soare A. Anastasia. 1997. Available at: http://www.anastasia.net

9 Sclafani AP, Jung M. Desired position, shape, and dynamic range of the normal adult eyebrow. Arch Facial Plast Surg 2010;12: 123-127

10 Biller JA, Kim DW. A contemporary assessment of facial aesthetic preferences. Arch Facial Plast Surg 2009;11:91-97

11 Wong BJ, Karimi K, Devcic Z, McLaren CE, Chen WP. Evolving attractive faces using morphing technology and a genetic algorithm: a new approach to determining ideal facial aesthetics. Laryngoscope 2008;118:962-974 